\title{
Personalidad Eficaz en Estudiantes Chilenos de Ingeniería de Primer Año
}

\author{
Mauricio I. González ${ }^{(1)}$, Pablo J. Castro ${ }^{(1)}$, María E. Martín ${ }^{(2)}$ \\ ${ }^{(1)}$ Depto. de Psicología, Universidad de La Serena. Matta 147, Coquimbo-Chile. \\ (e-mail: mgonzale@userena.cl; pablocastro@userena.cl) \\ ${ }^{(2)}$ Depto. de Psicología Evolutiva y de la Educación, Universidad Complutense de Madrid, \\ Facultad de Educación, Ciudad Universitaria 28040 - Madrid, España. \\ (e-mail: mariaeugeniamartin@edu.ucm.es)
}

Recibido Mar. 17, 2011; Aceptado May. 02, 2011; Versión final recibida May. 05, 2011

\begin{abstract}
Resumen
Se presenta un estudio sobre el constructo Personalidad Eficaz a partir de un cuestionario de personalidad eficaz en contextos educativos. Se analizan las propiedades psicométricas del cuestionario aplicado a una muestra de 450 estudiantes universitarios chilenos. Se obtuvo así un cuestionario con 25 ítems con una fiabilidad por consistencia interna de 0.88 , con cuatro factores que explican el $48 \%$ de la varianza total. En un análisis de segundo orden se obtiene un único factor que explica el $58 \%$ de la varianza total. Los resultados son consistentes con otros estudios realizados con muestras chilenas y españolas, avalan la solidez del constructo Personalidad Eficaz, y presentan evidencia sobre la posibilidad de contar con una sola versión del instrumento para estudios transculturales. Se concluye que es necesario complementar los resultados de estudios con capacidad predictiva para establecer la validez del instrumento para detectar a los estudiantes que tendrán dificultades de adaptación a la universidad.
\end{abstract}

\section{Efficient Personality in Chilean Students of Engineering of First Year}

\begin{abstract}
A study on the construct of Efficient Personality, based on a questionnaire on efficient personality in educational settings. The psychometric properties of the questionnaire, applied to a sample of 450 university students in Chile, were analysed. As a result, the questionnaire used consists of 25 items with an internal consistency of 0.88 , four factors which account for $48 \%$ of the total variance. In a second analysis, one unique factor was obtained accounting for $58 \%$ of the total variance. The results are consistent with other studies done with Chilean and Spanish samples which confirm the strength of the construct Efficient Personality. The results also present evidence on the possibility of relying on only one version of the instrument for transcultural studies. It is concluded that it is necessary to complement the results with studies of predictive capacity to establish the validity of the instrument to identify students who might suffer from adaptation difficulties in the university.
\end{abstract}

Keywords: efficient personality, efficient personality questionnaire, students of engineering 


\section{INTRODUCCIÓN}

El estudio de la relación entre atributos de la personalidad y la conducta eficaz, ya sea en los contextos académicos o laborales, ha tenido un importante desarrollo a través del constructo "Personalidad eficaz" operacionalizado a través de diferentes versiones del "Cuestionario de personalidad eficaz" que han sido reportadas desde el año 2004 hasta la fecha (Dapelo y Martín del Buey, 2006, 2007; Dapelo et al., 2006; Martín del Buey et al., 2008; Martín del Buey et al., 2004).

El desarrollo del constructo se fundamenta de forma preferente: en las investigaciones sobre autoeficacia de Bandura (Bandura, Jefrey y Gadjos, 1975); los planteamientos sobre inteligencia interpersonal e intrapersonal de Gardner $(1983,1999)$ y los estilos de pensamiento de Sternberg (1985); los estudios e investigaciones de Salovey y Mayer (1990) sobre la inteligencia emocional, y de forma más específica los descriptores del modelo de Inteligencia Emocional-Social de Bar-On (Reuven Bar-On, 2006); y el constructo de madurez basado en la competencia que se ha ido configurando a través de un largo periodo de años por los siguientes autores: por Heath (1976), Smith (1977), Vaillant y Milofsky, (1980), Crook, (1982), Waters y Sroufe (1983), Helson y Wink (1987), Kinney (1988), Steinberg et al. (1989), Garmez y Masten (1991) y Carpenter (1993).

En esa línea, se entiende a la persona eficaz como aquella que logra alcanzar sus metas dentro de plazos establecidos, gestionando sus propios recursos y los del medio (Dapelo y Martín del Buey, 2007). La Personalidad Eficaz se caracterizaría por un conjunto de capacidades y habilidades como, asertividad, autoestima, capacidad de trabajo, confianza en sí mismo, estabilidad emocional, estilo de pensamiento (capacidad de aprender de la experiencia, conciencia comprensiva, habilidad conceptual, habilidad para solucionar problemas, imaginación, visión de futuro, persuasión, versatilidad, visión realista del ambiente, viveza), extraversión, flexibilidad, independencia, iniciativa, locus de control, motivación de logro, optimismo, perseverancia, tolerancia a la incertidumbre, toma de riesgos, valores personales, etc. (Carbonero y Merino, 2004; Dapelo et al., 2006).

Martín del Buey y sus colaboradores $(1997,2000,2003,2008)$ definen la personalidad eficaz como un constructo teórico empírico compuesto por cuatro dimensiones en torno al yo. La primera dimensión fue denominada "Fortalezas del yo" y se relaciona con el autoconcepto y la autoestima. Esta dimensión es considerada el eje central de la personalidad eficaz. La Segunda dimensión se denominó, "Demandas del yo" y se relaciona con la motivación de logro, las atribuciones de control y las expectativas. La tercera dimensión se denomina "Retos del yo" y se relaciona con el afrontamiento de problemas y la toma de decisiones. La cuarta y última dimensión se denomina "Relaciones del yo" y se relaciona con la comunicación, la empatía y la asertividad que a su vez se relacionan con las habilidades sociales. Teóricamente se asume que las cuatro dimensiones funcionan de manera interactiva, influyéndose mutuamente, y complementándose (Martín del Buey et al., 2008).

Como se señaló inicialmente, se han reportado diversos estudios sobre el constructo a partir de escalas aplicadas a muestras con diferentes focos, por un lado, la personalidad eficaz para la adaptación a la escuela secundaria (Martín del Buey et al., 2004) y Por otro lado, para la adaptación profesional en el ámbito tanto universitario (Dapelo y Martín del Buey, 2006), como en el técnico (Martín del Buey et al., 2008). La cantidad de ítems de las escalas reportadas varían desde 60 de la escala original, hasta versiones de 23 o 24 ítems. Se ha aplicado a muestras de estudiantes españoles y chilenos de ambos sexos y como se señalaba anteriormente, de al menos dos grupos de edades diferentes (entre 16 y 18 en un caso y entre 18 y 22 en el otro).

Los resultados de estos estudios son consistentes en mostrar que los diferentes aspectos que conforman el constructo de "Personalidad eficaz", aparecen agrupados en cuatro esferas del yo, significativamente correlacionadas, dando así sustento empírico a la conceptualización de la personalidad eficaz como un constructo unitario global. También, es importante señalar que se ha conseguido una reducción importante en el número de ítems sin disminuir la confiabilidad global de la prueba y sin alterar de manera importante su estructura factorial, lo que permite contar con un instrumento más eficiente. 
Por otro lado, en el "Cuestionario de personalidad eficaz para la formación profesional" se encuentra el factor "autoestima" claramente diferenciado del factor "Autoconcepto social", lo que no se observa en las otras muestras de menor edad o menor nivel educativo, y este hallazgo se considera como evidencia para sostener una estructura multidimensional del autoconcepto (Martín del Buey et al., 2008). Además, está pendiente establecer la posibilidad de una única versión del instrumento que pueda ser aplicada de manera estándar a estudiantes de diferentes países y en un rango de edad, desde la educación secundaria (o parte de ella) hasta la formación universitaria.

En el marco de la formación universitaria, las facultades se han caracterizado por mantener altas tasa de reprobación, sobre todo en los primeros años (Abdala et al., 2008). Estas tasas de reprobación tienen como consecuencia el importante incremento de los años de duración del pregrado y el aumento de las tasas de deserción, que tiene un impacto en los proyectos de vida de los estudiantes y en los indicadores de las universidades. Para enfrentar este problema, en distintas universidades se han implementado diferentes programas remediales para disminuir el impacto negativo de la reprobación. Así, este estudio se enmarca en la búsqueda de factores de personalidad que permitan predecir el resultado académico de los estudiantes que ingresan a la facultad de ingeniería, más que en los factores cognitivos, y que puedan orientar una propuesta psicoeducativa que disminuya los riegos de deserción, disminuya los niveles de reprobación e incremente la calidad de vida del estudiante y futuro profesional (Castaño et al., 2006; Núñez y González, 1994).

En particular, el objetivo de este artículo es conocer las propiedades psicométricas del instrumento "Cuestionario de personalidad eficaz en contextos educativos" y determinar si estas son equivalentes a las reportadas por las investigaciones previas, en una muestra de estudiantes chilenos, que recién ingresan a la universidad, a estudiar una carrera de una Facultad de Ingeniería en una universidad estatal y regional. Los resultados constituirán un valioso aporte para la discusión sobre la consistencia, la pertinencia y la utilidad del constructo "Personalidad eficaz".

\section{MÉTODO}

\section{Participantes}

La muestra está compuesta por 450 estudiantes (315 hombres y 135 mujeres) con un promedio de 18 años de edad, que ingresaron a primer año a la facultad de ingeniería de una universidad de la Comuna de La Serena el año 2006.

\section{Instrumento}

Se utilizó la escala "Cuestionario de personalidad eficaz en contextos educativos" de 28 ítems, con un formato de respuesta tipo escala Likert, graduado de uno a cinco en un continuo que va desde "Total desacuerdo" a "Total acuerdo". La escala tiene tres factores de primer orden, Autorrealización Académica (ítems 1, 8, 10, 15, 19, 23, 30, 35, 38, 44, 57), Autorrealización Socio Afectiva (ítems 13, 18, 22, 27, 29, 31, 41, 42, 53, 56, 58) y Eficacia Resolutiva (ítems 11, 12, 16, 26,28 y 40 ) que explican el $47,42 \%$ de la varianza total y con un coeficiente alfa de Cronbach de .87 (Martín del Buey et al., 2004).

\section{Procedimiento}

El instrumento se aplicó a todos los estudiantes que ingresaron a la Facultad de Ingeniería el año 2005, durante el mes de marzo de ese año, de manera colectiva por asignatura. Fue aplicado por los profesores de las asignaturas del primer semestre siguiendo las instrucciones y coordinado por medio de la Dirección de Docencia de la universidad a la que pertenecían los alumnos. El instrumento se aplicó en su versión original sin realizar adaptaciones de lenguaje.

\section{Análisis estadísticos}

En primer lugar se realizó un análisis de la estructura factorial del instrumento a partir de los 28 ítems de la escala reportada por Martín del Buey et al. (2004), por medio de un análisis de componentes principales de primer orden con rotación oblicua "Oblimín" y un análisis de la confiabilidad por medio del coeficiente Alfa de Cronbach. Posteriormente se realizó un análisis de 
ítems para eliminar aquellos que tuvieran una correlación ítem total inferior a .25 y/o que su eliminación incrementara de manera considerable la consistencia interna de la escala. A continuación, una vez eliminados 2 ítems, se volvió a realizar el mismo análisis de componentes principales señalado y por último, se realizó un análisis de segundo orden con el mismo procedimiento.

\section{RESULTADOS Y DISCUSIÓN}

A partir del primer análisis con los 28 ítems de la escala reportada por Martín del Buey et al. (2004), se encontró una confiabilidad por consistencia interna de .85 y utilizando los mismos procedimientos reportados en el artículo antes citado (análisis de componentes principales con rotación oblicua Oblimin), se extrajeron 3 factores para comparar el ajuste de los ítems a la estructura inicial. Los tres factores explican el 39.8\% de la varianza total y la correspondencia de los reactivos que cargan en cada componente es muy buena, considerando que solo dos de ellos no cargan en los componentes esperados (tabla 1). En un análisis de componentes principales de segundo orden, los tres componentes se agrupan en uno y explican el $61.2 \%$ de la varianza total.

Después de la realización del segundo procedimiento, por medio de un análisis de ítem, se eliminaron dos reactivos que mantenían una correlación ítem total inferior a .25 (9 y 19) y uno que si bien tenía un coeficiente de correlación ítem total superior al criterio señalado, su eliminación incrementaba de manera importante la consistencia interna del instrumento (24). Una explicación para lo que ocurrió con este ítem es a partir de las diferencias culturales entre los estudiantes chilenos y españoles sobre la valoración del estudio y su relación con las notas, que en el caso de los estudiantes chilenos consideran que se debe estudiar para aprender y no solo para sacarse "buenas notas". El instrumento quedó conformado por 25 ítems con una fiabilidad (consistencia interna) de .88.

\section{Estructura factorial}

Al aplicar un Análisis de Componentes Principales con rotación oblicua "Oblimin", se constató que todos los ítems obtuvieron comunalidades superiores a .30, lo que si bien es un valor bajo, en los análisis de escalas tipo Likert se considera suficiente. De acuerdo a los antecedentes teóricos y a la configuración del gráfico de sedimentación, se determinó extraer cuatro factores debido a que a partir del componente 5 el aporte adicional de varianza explicada es pequeño (figura 1). Como criterio de saturación significativo del ítem en cada componente, se consideró un peso factorial de .30. Los cuatro componentes extraídos explican un 48. $67 \%$ de la varianza total (Tabla 2).

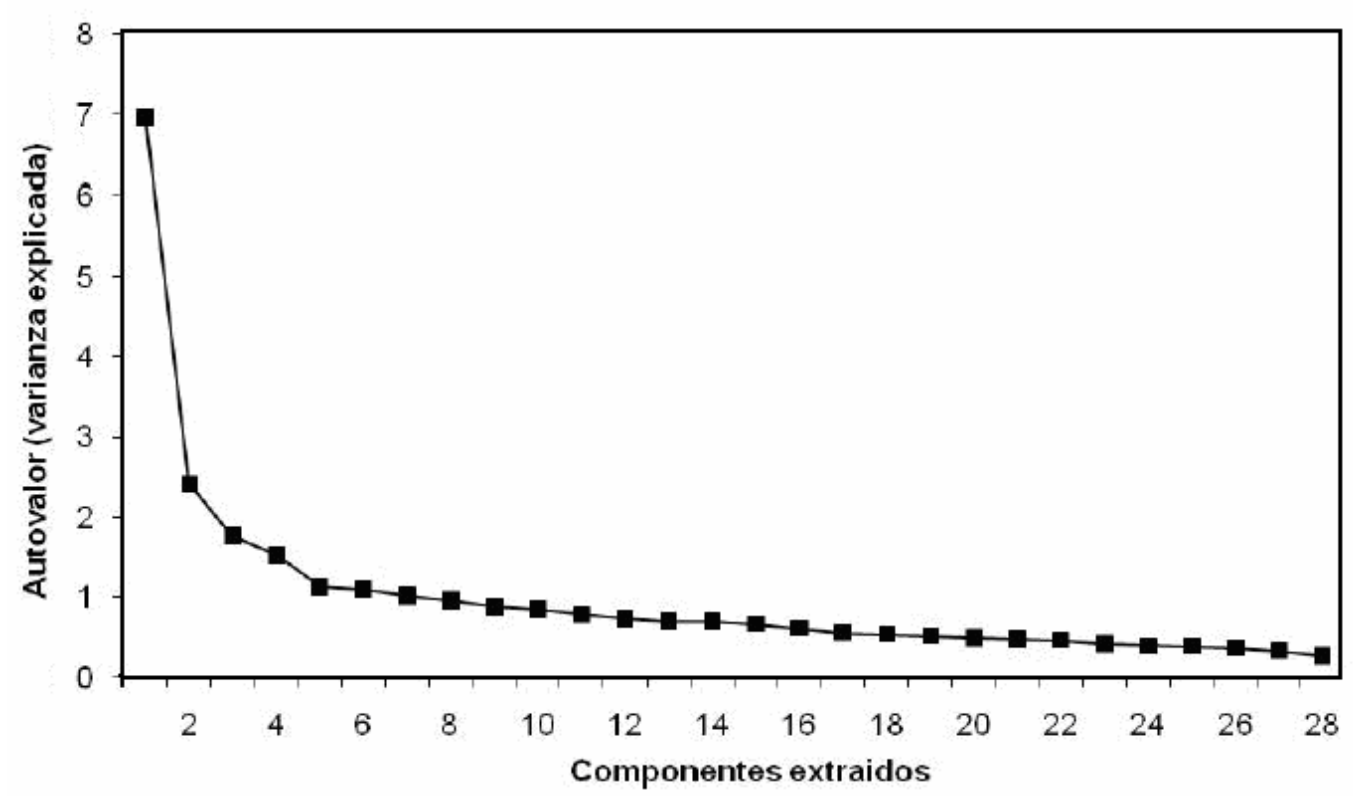

Fig. 1: Autovalores de los componentes extraídos en el análisis de componentes principales 
Tabla 1: Análisis Factorial de la Escala Original (Martín del Buey et al., 2004). En la Tabla, AA = Autorrealización académica, ASA = Autorrealización Socioafectiva, $\mathrm{ER}=$ Eficacia Resolutiva. $\mathrm{EI}$ signo $\left(^{\star}\right)$ indica ítems que no cargan en los factores esperados de acuerdo a los estudios previos

\begin{tabular}{|c|c|c|c|}
\hline & \\
\hline & $A A$ & ASA & ER \\
\hline 7. Creo que tendré éxito en mis estudios futuros. & 0.70 & 0.33 & \\
\hline 3. Creo que aprobaré todas las asignaturas de este semestre. & 0.69 & & \\
\hline 2. Estudio porque me interesa aprender cosas nuevas. & 0.61 & & -0.36 \\
\hline 12. Estudio porque me gusta superar retos. & 0.60 & & -0.44 \\
\hline 1. Me considero un buen estudiante. & 0.60 & & -0.34 \\
\hline 28. Creo que puedo encontrar una solución a cualquier problema que se me plantee. * & 0.59 & & \\
\hline 20. Mis éxitos en los estudios se deben a mi esfuerzo. & 0.57 & & -0.48 \\
\hline 17. Mis éxitos en los estudios se deben a mi capacidad. & 0.54 & & -0.31 \\
\hline 10. Nadie me obliga a estudiar, estudio porque quiero hacerlo. & 0.53 & & -0.32 \\
\hline 27. Estudio porque me gusta comprobar que soy capaz de hacerlo. & 0.51 & & -0.47 \\
\hline 19. Creo que seré incapaz de aprobar todas las asignaturas de este curso. & 0.39 & & \\
\hline 11. Hago amigos con facilidad. & & 0.78 & \\
\hline 25. Creo que tendré éxito en mis relaciones con los demás. & & 0.75 & \\
\hline 23. Mis éxitos en las relaciones con los demás se deben a mi habilidad para hacer amigos. & & 0.68 & \\
\hline $\begin{array}{l}\text { 14. Mis fracasos en las relaciones con los demás se deben a mi falta de habilidad } \\
\text { para hacer amigos. }\end{array}$ & & 0.65 & \\
\hline 26. Creo que soy una persona valiosa para los otros. & 0.37 & 0.58 & \\
\hline 22. Estoy a gusto con mi aspecto físico. & 0.43 & 0.56 & \\
\hline 18. En general me siento satisfecho conmigo mismo. & 0.51 & 0.52 & \\
\hline 6. Me acepto tal y como soy, con mis cualidades y defectos. & 0.42 & 0.50 & \\
\hline 16. Creo que me expreso con claridad. & 0.40 & 0.44 & \\
\hline 9. Mis fracasos en las relaciones con los demás se deben a que no les gusto. & & 0.35 & \\
\hline 8. Para tomar decisiones reúno toda la información que puedo encontrar. & & & -0.69 \\
\hline 15. Cuando tengo que tomar una decisión planifico cuidadosamente lo que voy a hacer. & & & -0.68 \\
\hline 5. Analizo las posibles consecuencias de mis decisiones antes de tomarlas. & & & -0.67 \\
\hline 21. Cuando tengo un problema dedico tiempo y esfuerzo para resolverlo. & 0.44 & & -0.58 \\
\hline 13. Cuando tengo un problema intento aprender de esa experiencia. & 0.32 & & -0.51 \\
\hline 4. Cuando tengo un problema trato de ver el lado positivo de la situación. & & & -0.45 \\
\hline 24. Estudio para sacarme buenas notas * & & & 0.37 \\
\hline
\end{tabular}

\section{Análisis de los componentes}

En la tabla 3 se muestra la matriz de relaciones de los reactivos con los componentes según su carga factorial. Todos los ítems tuvieron una carga factorial superior a .30 en al menos un componente. El primer componente quedó conformado por siete ítems (1, 2, 10, 12, 20, 27, 28), todos pertenecientes al componente originalmente denominado Autorrealización Académica. Los seis primeros ítems se relacionan directamente con la motivación por el estudio y el autoconcepto como estudiante y solo el ítem 28 aparece relacionado con un ámbito diferente de un nivel de generalidad mayor, pero relacionado con la autorrealización. Se considera pertinente mantener el nombre original de "Autorrealización académica" a este componente. El segundo componente quedó conformado por seis ítems $(11,14,16,23,25,26)$, los que en su totalidad pertenecen al componente denominado "Autorrealización socioafectiva" de la escala original. 
Cinco de los seis ítems se relacionan con la autopercepción de la capacidad para iniciar y mantener exitosamente relaciones de amistad, un ítem (26) se relaciona con la autopercepción del propio valor personal y un ítem (16) se refiere concretamente a la autopercepción de la capacidad de expresarse con claridad. Por lo anterior se propone conservar la denominación de "Autorrealización socioafectiva" de la versión inicial.

Tabla 2: Varianza Explicada por los Factores

\begin{tabular}{|l|l|c|c|}
\hline & \multicolumn{1}{|c|}{ Total } & $\begin{array}{c}\text { \% de la varianza } \\
\text { \% de la varianza }\end{array}$ & \% acumulado \\
\hline & Total & 27.3 & 27.3 \\
\hline 2 & 6.81 & 9.2 & 36.5 \\
\hline 3 & 1.61 & 6.5 & 42.9 \\
\hline 4 & 1.43 & 5.7 & 48.7 \\
\hline
\end{tabular}

Tabla 3: Análisis Factorial de la Escala Reducida (25 ítems)

\begin{tabular}{|c|c|c|c|c|}
\hline & 1 & 2 & 3 & 4 \\
\hline 2. Estudio porque me interesa aprender cosas nuevas. & 0.77 & & & \\
\hline 12. Estudio porque me gusta superar retos. & 0.71 & & 0.33 & \\
\hline 27. Estudio porque me gusta comprobar que soy capaz de hacerlo. & 0.63 & & 0.38 & \\
\hline 10. Nadie me obliga a estudiar, estudio porque quiero hacerlo. & 0.62 & & & \\
\hline 1. Me considero un buen estudiante. & 0.60 & & & -0.38 \\
\hline 20. Mis éxitos en los estudios se deben a mi esfuerzo. & 0.57 & & 0.45 & -0.34 \\
\hline $\begin{array}{l}\text { 28. Creo que puedo encontrar una solución a cualquier problema que se me } \\
\text { plantee. }\end{array}$ & 0.49 & & & -0.48 \\
\hline 11. Hago amigos con facilidad. & & 0.87 & & \\
\hline $\begin{array}{l}\text { 23. Mis éxitos en las relaciones con los demás se deben a mi habilidad para } \\
\text { hacer amigos. }\end{array}$ & & 0.79 & & \\
\hline 25. Creo que tendré éxito en mis relaciones con los demás. & & 0.76 & & -0.36 \\
\hline $\begin{array}{l}\text { 14. Mis fracasos en las relaciones con los demás se deben a mi falta } \\
\text { de habilidad para hacer amigos. }\end{array}$ & & 0.63 & & \\
\hline 26. Creo que soy una persona valiosa para los otros. & & 0.53 & & -0.45 \\
\hline 16. Creo que me expreso con claridad. & 0.39 & 0.45 & & -0.31 \\
\hline 5. Analizo las posibles consecuencias de mis decisiones antes de tomarlas. & & & 0.74 & \\
\hline $\begin{array}{l}\text { 15. Cuando tengo que tomar una decisión planifico cuidadosamente lo que } \\
\text { voy a hacer. }\end{array}$ & & & 0.74 & \\
\hline 8. Para tomar decisiones reúno toda la información que puedo encontrar. & 0.38 & & 0.69 & \\
\hline 21. Cuando tengo un problema dedico tiempo y esfuerzo para resolverlo. & & & 0.57 & \\
\hline 13. Cuando tengo un problema intento aprender de esa experiencia. & & & 0.55 & \\
\hline 4. Cuando tengo un problema trato de ver el lado positivo de la situación. & & & 0.53 & \\
\hline 6. Me acepto tal y como soy, con mis cualidades y defectos. & & 0.32 & & -0.74 \\
\hline 18. En general me siento satisfecho conmigo mismo. & & 0.36 & & -0.71 \\
\hline 22. Estoy a gusto con mi aspecto físico. & & 0.41 & & -0.70 \\
\hline 7. Creo que tendré éxito en mis estudios futuros. & 0.57 & & & -0.60 \\
\hline 3. Creo que aprobaré todas las asignaturas de este semestre. & 0.52 & & & -0.54 \\
\hline 17. Mis éxitos en los estudios se deben a mi capacidad. & 0.41 & & 0.31 & -0.53 \\
\hline
\end{tabular}


El tercer componente está conformado por seis ítems $(4,5,8,13,15,21)$, todos pertenecientes al componente anteriormente denominado "Eficacia resolutiva". Los seis ítems tienen en común que se refieren a la manera como la persona toma decisiones y resuelve los problemas que se le presentan. No hacen alusión a cuán eficaz es la persona, pero sí a la cantidad de tiempo, planificación, meticulosidad y disposición anímica con que enfrenta las decisiones y los problemas. Considerando lo anterior, consideramos que la denominación más adecuada para este componente es de "Afrontamiento de problemas" El cuarto componente está conformado por seis ítems $(3,6,7,17,18,22)$ de los cuales tres $(3,7,17)$ provienen del componente previo denominado "Autorrealización académica" y los otros tres $(6,18,22)$ provienen de "Autorrealización socioafectiva". Los tres primeros se relacionan con la autoestima y los últimos se relacionan, dos con las expectativas de éxito futuro y uno con la atribución de éxito a las propias capacidades. Resulta consistente denominar a este componente como "Autoestima y autorrealización".

La fiabilidad de los cuatro componentes fluctúa entre un mínimo de .74 y un máximo de .79 (Tabla 4). La fiabilidad obtenida es alta, no obstante, en la versión anterior se obtienen valores levemente superiores aunque más heterogéneos. Los coeficientes de correlación entre cada componente y la escala total son fuertes y positivos a la vez que significativos al nivel alfa de .00 (tabla 5). Los coeficientes encontrados muestran el constructo medido es homogéneo y probablemente unitario respecto de sus dimensiones. La fuerte correlación entre los componentes muestra que el análisis individual de cada uno de ellos, no aportaría información adicional o diferente a la obtenida por el puntaje total. Se realizó un análisis de componentes principales de segundo orden, se obtuvo un solo factor que explica el $58 \%$ de la varianza total y los pesos y la carga de cada componente inicial en el componente de segundo orden, fluctuó entre .69 y .82.

Las propiedades psicométricas de esta aplicación de la Escala resultan claramente similares a la escala original española aplicada a adolescentes. La confiabilidad por consistencia interna obtenida de .85, solo es levemente inferior a la reportada por Martín del Buey et al. (2004) que fue de .87. Además, se configuran prácticamente los mismos tres componentes que en dicha escala y en un análisis factorial de segundo orden los tres se funden en un solo componente que explica el $61.24 \%$ de la varianza total mientras que en el artículo referido se obtiene un $47.43 \%$.

Tabla 4: Fiabilidad de los Componentes de Primer Orden

\begin{tabular}{|l|c|c|}
\hline Componentes & No de ítem & Coef. Alfa \\
\hline Autorrealización Académica & 7 & .79 \\
\hline Autorrealización Socioafectiva & 6 & .78 \\
\hline Afrontamiento de Problemas & 6 & .74 \\
\hline Autoestima y autorrealización & 6 & .76 \\
\hline Fiabilidad Total & 25 & .88 \\
\hline
\end{tabular}

Tabla 5: Correlaciones entre los Componentes y el Total

\begin{tabular}{|l|c|l|}
\hline Componentes & Escala Total & Componentes \\
\hline Autorrealización Académica & $0.80^{*}$ & Autorrealización Académica \\
\hline Autorrealización Socioafectiva & $0.71^{*}$ & Autorrealización Socioafectiva \\
\hline Afrontamiento de Problemas & $0.72^{\star}$ & Afrontamiento de Problemas \\
\hline Autoestima y autorrealización & $0.80^{*}$ & Autoestima y autorrealización \\
\hline${ }^{*} p<0.000$ & & \\
\hline
\end{tabular}


Tabla 6: Carga Factorial de Componentes de Primer Orden y

Componente Unitario de Segundo Orden

\begin{tabular}{|l|c|}
\hline Componente & carga \\
\hline Autorrealización Socio Afectiva & 0.69 \\
\hline Autorrealización Académica & 0.81 \\
\hline Afrontamiento de Problemas & 0.73 \\
\hline Autoestima y autorrealización & 0.82 \\
\hline
\end{tabular}

Solo dos ítems no cargan en los componentes esperados y considerando que la escala se aplicó sin hacer una adaptación previa, es posible suponer que en el caso del ítem "Estudio para sacarme buenas notas" la afirmación tenga un significado diferente y más negativo en la cultura chilena. Este ítem posteriormente fue eliminado y al revisar los reportes de estudios posteriores se observa que también se eliminó en ellos. El otro ítem, "creo que puedo encontrar una solución a cualquier problema que se me plantee" tendría que haber cargado en el componente de Autorrealización Socioafectiva, pero carga en Autorrealización Académica y se puede explicar porque culturalmente el ítem se entendió referido a problemas académicos.

Considerando que posteriormente se ha seguido reportando estudios con nuevas aplicaciones del "Cuestionario de personalidad eficaz en contextos educativos", se realizó un nuevo análisis, previa eliminación de tres ítems por medio de un análisis de ítems. La estructura de componentes encontrada en este estudio es muy similar a la reportada por Dapelo et al. (2006), en una muestra de adolescentes chilenos de tercer y cuarto año de Enseñanza media (entre 16 y 18 años). El $64 \%$ de los ítems están redactados de la misma manera, el resto son sobre aspectos diferentes dentro de un mismo dominio. Por lo mismo, resulta llamativo que emergieran los mismos componentes con una muy similar distribución de ítems. Por otro lado, se observan diferencias con la estructura reportada por Martín del Buey et al. (2008) a partir de una muestra de estudiantes de centros de formación profesional españoles, de edades entre 18 y 22 años, en la cual obtuvieron 6 componentes con la autoestima claramente diferenciada en un componente aislado. Por una parte, los resultados con muestras chilenas son más similares entre sí a pesar de las diferencias en las edades, en el contexto de formación y de ser de regiones diferentes de Chile (cuarta y quinta regiones). Por otro lado, respecto a la comparación con la muestra española, se podría decir que hay mayor similitud en las edades y en el contexto de formación.

Junto con lo anterior, están las diferencias culturales obvias que podrían explicar las diferencias en la cantidad de factores encontrados, sobre todo en la emergencia de la autoestima como un componente diferenciado. No obstante, no hay que descartar la edad como una explicación alternativa ya sea por sí sola o en interacción con la cultura, que podría explicar esta diferencia, ya que la muestra de estudiantes de primer año de universidad es en general una muestra que aún se encuentra en el rango etario de la adolescencia. Cabe destacar que tanto en el presente estudio como en los demás reportes citados se ha encontrado, de manera consistente, coherencia entre los datos y la teoría en cuanto al modelo conceptual que sustenta el constructo de "Personalidad eficaz" en cuatro esferas del yo altamente interrelacionadas.

En este estudio, el instrumento se aplicó con el propósito principal de evaluar la posibilidad de detectar tempranamente en estudiantes que ingresan a la universidad factores de riesgo de fracaso, en el ámbito de la personalidad y la autoestima. Por lo mismo no se ha diseñado este estudio para establecer comparaciones entre diferentes muestras. Teniendo esto presente, ya que implica algunas limitaciones de diseño para la validez de las comparaciones realizadas, los hallazgos resultan en un nuevo respaldo a la consistencia entre la teoría y los datos empíricos obtenidos a través de diferentes estudios. También, a partir de dichas aproximaciones es posible acercarse al consenso y elaboración de un sólo instrumento que pueda ser utilizado tanto en adolescentes como en educación superior y en diferentes países de habla hispana. 
Independientemente de los resultados hasta ahora obtenidos, está pendiente evaluar la validez referida a criterio del instrumento. Se ha señalado en estudios previos la posibilidad de utilizar algunas escalas de autoeficacia (Carrasco y del Barrio, 2002; Sanjuán y Bermúdez, 2000) que podrían ser buenos criterios concurrentes y además se requiere analizar la validez predictiva de la escala. Respecto al último aspecto, con los datos de la aplicación de la escala en la muestra de estudiantes que ingresaron a primer año a la facultad de ingeniería, se está realizando la segunda parte de este estudio que pretende correlacionar el resultado académico de los estudiantes, desde el primer año hasta el último, incluyendo la información de quienes reprobaron y de quienes abandonaron la carrera. De esta manera podremos saber si el cuestionario, permitiría identificar a aquellos estudiantes que presenten más riesgo y poder realizar las acciones remediales 0 preventivas pertinentes.

\section{CONCLUSIONES}

De los resultados obtenidos, de su análisis y de su discusión, se pueden obtener las siguientes conclusiones, sobre la aplicación a esta muestra del "Cuestionario de Personalidad Eficaz en Contextos Educativos":

1) El cuestionario Personalidad eficaz aplicado a una muestra de estudiantes de ingeniería de primer año mantiene la confiabilidad y la validez de estudios previos;

2) El constructo personalidad eficaz ha sido consistente en su aplicación para describir diferentes muestras de estudiantes de enseñanza media y universitarios;

3) Es posible obtener una versión única para aplicar en España y Chile y probablemente en otros países latinoamericanos; y

4) Es necesario complementar estos resultados con estudios de validez predictiva para establecer la capacidad del instrumento para detectar a los estudiantes que tendrán dificultades de adaptación a la universidad.

\section{REFERENCIAS}

Abdala, S., Castiglione, A.M. e Infante, L. M., La deserción universitaria. Una asignatura pendiente para la gestión institucional. Cuadernos FhyCs-UNJu, 34, 173-191. (2008).

Bandura, A., Self-efficacy: Towards a unifying theory of behavioral change. Psychological Review, 84, 191-215 (1977).

Bandura, A., Pensamiento y acción. Fundamentos sociales. Barcelona: Martínez Roca (1987).

Bandura. A., The reconstrual of "free will" from the agentic perspective of social cognitive theory. En J. Baer, J.C. Kaufman y R.F. Baumeister (Eds.), Are We Free? Psychology and Free Will. (pp. 86-127). Oxford: Oxford University Press (2008).

Bandura, A., Jeffery, RW y Gadjos, E., Generalizing change through participant modeling with selfdirected mastery. Behavioral Research and Therapy 13, 141-152 (1975).

Bar-On, R., The Bar-On model of emotional-social intelligence (ESI). Psicothema, 18, supl., 13-25 (2006).

Carbonero, M.A. y Merino Tejedor, E., Autoeficacia y madurez vocacional. Psicothema, 16(2), 229234 (2004).

Carrasco Ortiz, M.A., y Del Barrio Gándara, M.V., Evaluación de la autoeficacia en niños y adolescentes. Psicothema, 14(2), 323-332 (2002). 
Castaño, E., Gallón, S., Gómez, K., y Vásquez, J., Análisis de los factores asociados a la deserción y graduación estudiantil universitaria. Lecturas de Economía Universidad de Antioquia, 65, 9- 36 (2006).

Carpenter, B.N., Relational competence. En D. Perman y W.H. Jones (Eds.), Advances in personal relationships. Londres: Jessica Kingsley Publishers (1993).

Crook, R.H., An investigation of the relationship between psychological development and self steem, ego identity, vocational maturity, academic achievement and work achievement. Dissertation Abstracts International, 43/O4B (1982).

Dapelo, B., Marcone Trigo, R., Martín del Buey, F., Martín Palacio, E., y Fernández Zapico, A., Adaptación chilena del Cuestionario de Personalidad Eficaz para Adolescentes. Psicothema, 18(1), 130-134 (2006).

Dapelo, B. y Martín del Buey, F., Estudiante eficaz en el contexto de formación de profesores. Adaptación del cuestionario de personalidad eficaz en el ámbito universitario. Revista de Orientación Educacional, 20(38), 65-79 (2006).

Dapelo, B. y Martín del Buey, F., Personalidad eficaz en el contexto de la educación técnico profesional. Revista de Orientación Educacional, 21 (39), 13- 29 (2007).

Gardner, H., Frames of Mind. The Theory of Multiples Intelligences. Nueva York, Basic Books. (versión castellana (2001): estructuras de la mente. La teoría de las inteligencias múltiples. México, FCE) (1983).

Gardner, H., Intelligence Reframed: Multiple Intelligences for the 21st Century. Nueva York, Basic Books. (Versión castellana: (2001) La inteligencia reformulada. Las inteligencias múltiples en el Siglo XXI. Barcelona Paidós (1999).

Garmez, M. y Masten, A.S., The protective role of competence indicators in children at risk. En M. Cummings, A.L. Greene y K.H. Karraker (Eds.): Life-span development psychology: Perspectives on stress and coping. Hillsdale: LEA (1991).

Heath, D.H., Maturity and competence: a transcultural view. N.Y.: Gardner Press (1977).

Helson, R. y Wink, P., Two conceptions of maturity examined in the findings of a longitudinal study. Journal of Personality and Social Psychology, 53, 531-541 (1987).

Kinney, B.A., Individuation in women during late adolescence and early adulthood as a function on psychological maturity and interpersonal competence. Dissertation Abstracts International, 50/OB2 (1988).

Martín del Buey, F., Programa integrado de acción tutorial. Valparaíso: Revista de Orientación Educacional, 19-20, 71-88 (1997).

Martín del Buey, F., Programa integrado de acción tutorial: marco conceptual. Desarrollo de la personalidad eficaz en contextos educativos. Oviedo: Ediciones fmb2001 (2000).

Martín del Buey, F., y Fernández Zapico, A., Programa de desarrollo de la personalidad eficaz en contextos educativos: primeros resultados. Magister, 19, 277-291 (2003).

Martín del Buey, F., Martín Palacio, E., Fernández Zapico, A., Dapelo Pellerano, B. y Marcone Trigo, R., Evaluación de la personalidad eficaz en contextos educativos: primeros resultados. Chile: Revista de Orientación Educacional, 33-34, 79-101 (2004). 
Martín del Buey, F., Fernández Zapico, A., Martín Palacio, E., Dapelo Pellerano, B., Marcone Trigo, R., y Granados Urban, P., Cuestionario de personalidad eficaz para la formación profesional. Psicothema, 20(2), 224-228 (2008).

Núñez, C., y González, J., Determinantes del rendimiento académico. Servicio de Publicaciones de la Universidad de Oviedo (1994).

Salovey, P. y Mayer, J.D. Emotional intelligence. Imagination, Cognition, and Personality, 9, 185211, (1990).

Sanjuán, P., Pérez, A, y Bermúdez, J., Escala de autoeficacia general: datos psicométricos de la adaptación para población española. Psicothema, 12, Suplemento, 509-513 (2000).

Sternberg, R.J., Beyond IQ: a triarchic theory of human inteligence. Cambridge University Press (1985).

Steinberg, L., Elmen, J.D. y Mounts, N.S., Authoritative parenting, psychosocial maturity and academic success among adolescents. Child development, 60, 1424-1436 (1989).

Vaillant, G.E. y Milofsky, E., Natural history of male psychological health. American Journal of Psychiatry, 1348-1359 (1980).

Waters, E. y Sroufe, L.A., A developmental perspective on competence. Developmental Review, 1, 59-79, (1983). 
